\title{
Chinese herbal medicine Tangshen Formula treatment for type 2 diabetic kidney disease in the early stage: study protocol for a randomized controlled trial
}

\author{
De Jin ${ }^{1}$, Wen-Jing Huang ${ }^{1}$, Xiang Meng ${ }^{2}$, Fan Yang ${ }^{2}$, Qi Bao ${ }^{1}$, Mei-zhen Zhang ${ }^{1}$, Ya-nan Yang ${ }^{2}$, Qing $\mathrm{Ni}^{1 *}$, \\ Feng-Mei Lian ${ }^{1 *}$ and Xiao-Lin Tong ${ }^{1 *}$
}

\begin{abstract}
Background: Diabetic kidney disease (DKD) is the main cause of end-stage kidney disease and has become a heavy economic and social burden due to its high prevalence and morbidity. The most effective strategy is that patients with DKD should be diagnosed and treated early. Preliminary studies showed that the Chinese herbal Tangshen Formula (TSF) may delay the progression of DKD, reducing microalbuminuria and macroalbuminuria and improving renal function. We designed a randomized, double-blind, placebo-controlled trial to evaluate the efficacy of TSF in patients with DKD.

Methods/design: This trial is a 13-center, randomized, double-blind, placebo-controlled study. A total of 632 participants will be randomized in a 1:1 ratio to an experiment group (TSF plus losartan) and a control group (placebo plus losartan). The trial cycle will last 24 weeks. The primary outcome will be the change in the urine microalbumin-creatinine ratio from baseline to week 24. The secondary outcome will be the change in the rate of progression to the clinical proteinuria period after intervention, the rate of urine microalbumin negative conversion, the rate of normal urinary microalbumin, the doubling rate of the baseline creatinine value and the glomerular filtration rate between the two groups. Safety in medication will also be evaluated.
\end{abstract}

Discussion: We hypothesize that patients with type 2 diabetes in the early stage of DKD will benefit from TSF. If successful, this study will provide evidence-based recommendations for clinicians.

Trial registration: ClinicalTrials.gov, NCT03009864. Registered January 2017.

Keywords: Diabetic kidney disease, Traditional Chinese medicine, Treatment, Randomized controlled trial

\section{Background}

Diabetic kidney disease (DKD) is the most common diabetic microvascular complication and the primary cause of end-stage kidney disease (ESKD). Pooled data from 54 countries revealed that more than $80 \%$ of ESKD arises from diabetes, hypertension or a combination of both. The prevalence of ESKD was also up to 10 times higher in people with diabetes than in those without [1]. Several

\footnotetext{
*Correspondence: niqing669@163.com; 694397644@qq.com; tongxiaolin66@sina.com

${ }^{1}$ Guang'anmen Hospital, Chinese Academy of Chinese Medical Sciences, Beijing 100053, China

Full list of author information is available at the end of the article
}

studies showed that $20 \%$ of people with diabetes from the UK [2] and 40\% of individuals with diabetes in the USA will develop chronic kidney disease (CKD), whereas $19 \%$ show signs of DKD stage 3 or higher [3]. It is responsible for $40 \%$ of patients experiencing dialysis after diagnosis of diabetes mellitus in the developed world [4]. Moreover, there has also been a continuous increase in the incidence of ESKD due to the increasing incidence of T2DM, notably in China, with $11.9 \%$ from the high incidence of type 2 diabetes [5]. As impairment in renal function progresses, it is bound up with a high risk of mortality, cardiovascular events and hospitalizations [1] and is associated with a heavy economic burden, with

(C) The Author(s). 2019 Open Access This article is distributed under the terms of the Creative Commons Attribution 4.0 International License (http://creativecommons.org/licenses/by/4.0/), which permits unrestricted use, distribution, and 
estimated average annual healthcare costs ranging from $\$ 4573$ to $\$ 10,322$ [6]. Some risk factors of DKD have been identified, such as ageing, hypertension and hyperglycemia [7, 8]. Current strategies for DKD aim to delay deterioration of renal function through actively controlling glucose, blood pressure and blood lipid levels, and via restraint of the renin-angiotensin-aldosterone system (RAAS) [6-8], such as using angiotensin-converting enzyme inhibitors (ACEIs) and angiotensin II receptor blockers (ARBs).

However, these therapies are difficult to achieve in all patients with DKD and stable results in the long term as well as these treatments may not reverse DKD $[9,10]$, and even with treatment using currently effective therapies, ESKD will finally occur in a proportion of patients, requiring a dialysis regimen and kidney transplant. Therefore, the most effective strategy is that kidney disease in patients with diabetes should be diagnosed and treated early. Patients with type 2 diabetes with microalbuminuria are at high risk of progression to overt renal disease, indicating that those patients easily enter the stage of clinical diabetic nephropathy (stage 4 or 5). In addition, not only ESKD but also premature cardiovascular events are predicted by microalbuminuria in diabetes [11-13], whereas effective intervention therapies for microalbuminuria could improve clinical outcomes in DKD.

Although ACEIs or ARBs showed evidence for decreasing microalbuminuria in patients with type 2 diabetes and nephropathy $[14,15]$, some adverse events (cough, rhinitis, hyperkalemia, acute kidney injury and angioedema) related to ACEIs or ARBs might cause poor adherence [1-16]. Therefore, it is imperative to seek effective renoprotective therapies.

Chinese herbal medicine (CHM) has long been widely used in China [17]. Numerous studies have demonstrated the biological activity and therapeutic mechanism of CHM [18-21]. Recent studies show that certain Chinese herbs have renoprotective effects, improving the glomerular filtration rate (GFR) and decreasing proteinuria, especially in patients with microalbuminuria [2226]. This study is designed to investigate whether Tangshan Formula (TSF) may represent a potential remedy for slowing disease progression in early type 2 diabetic nephropathy. If positive, this work may provide an evidence-based medicine remedy for slowing or preventing the clinical progression of DKD.

\section{Methods/design}

\section{Study design}

This protocol will be designed as a randomized, placebocontrolled and multicenter trial. Participants, investigators and statisticians will be blinded. A total of 632 subjects will be recruited at the following 13 tertiary A hospitals in mainland China: Guang An Men Hospital of the China Academy of Chinese Medical Sciences, The First Affiliated Hospital of Anhui University of Traditional Chinese Medicine, Beijing Hospital of Traditional Chinese Medicine, Hubei Hospital of Traditional Chinese Medicine, Zouping County Hospital of Traditional Chinese Medicine, Zibo Wanjie Hospital and Shijiazhuang Hospital of Traditional Chinese Medicine, Zhengzhou City Hospital of Traditional Medicine, Xingtai City Hospital of Traditional Medicine, Shexian Country Hospital of Traditional Medicine, Baoding City of Hospital of Traditional Medicine, Yantai Bai shi Hospital of Traditional Medicine and Jilin Province Hospital of Traditional Medicine. The trial will be implemented base on the principles of good clinical practice and reported according to the CONSORT statement [27, 28]. The trial flow diagram is illustrated in Fig. 1. The Standard Protocol Items: Recommendations for Interventional Trials (SPIRIT) [29] Checklist is shown in Additional file 1. This study has been registered at ClinicalTrials.gov (NCT03009864).

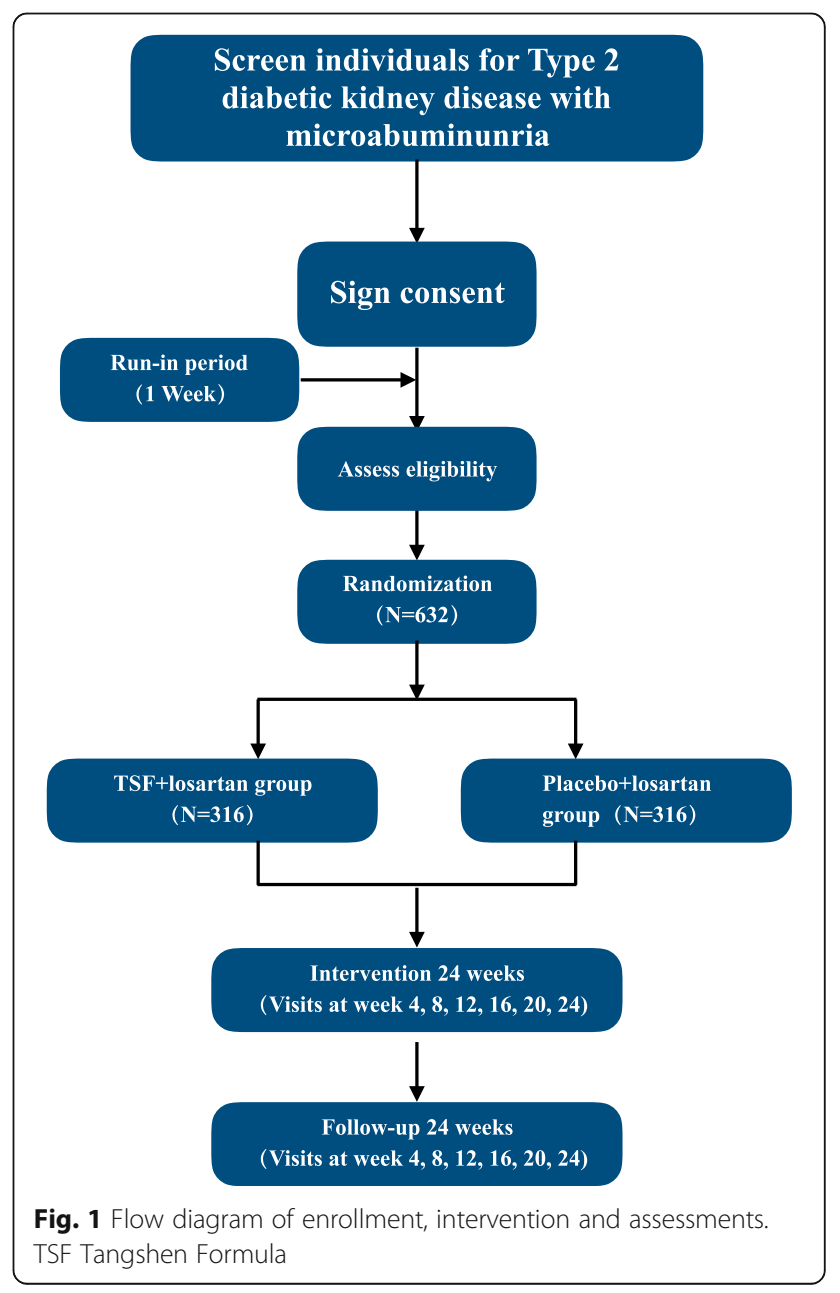




\section{Participants \\ Diagnostic criteria}

The diagnostic criteria for this trial will be set based on the American Diabetes Association guidelines [7], Chinese Diabetes Society guidelines [8], National Kidney Foundation Kidney Disease Outcomes Quality Initiative (NKF-KDOQI) guidelines [30] and Kidney Disease Improving Global Outcomes (KDIGO) 2012 Clinical Practice Guideline [31]. For a diagnosis of early-stage DKD, patients with DM will have to present the following conditions:

1. Diagnosed with type 2 diabetes

2. Patients with type 2 diabetes with microalbuminuria and urinary microalbumin excretion rate (UAER)

(a) For microalbuminuria, two repeated tests performed within 6 months will need to produce abnormal results

(b) UAER of 30-300 mg/24 h or urinary albuminto-creatinine ratio (ACR) of $30-300 \mathrm{mg} / \mathrm{g}(\mathrm{mg} /$ $\mathrm{mmol}$ )

3. Have clinical manifestations of kidney diseases, such as edema, anemia, renal dysfunction, etc.

\section{Inclusion criteria}

1. Patient diagnosed with DKD with microalbuminuria

2. Age $30-70$ years

3. Signed informed consent

\section{Exclusion criteria}

1. Proteinuria caused by non-DKD in the patient, such as gout, primary hypertension, tumors and proteinuria caused by chronic kidney disease

2. Cardiovascular, liver, kidney, hematopoietic system or other primary severe disease; serum transaminase more than double the standard value; serum creatinine $(\mathrm{SCr})$ higher than the upper limit of normal; and psychiatric patients

3. Pregnancy, preparation for pregnancy or lactation, or any history of drug allergy

4. The patient developed renal failure (anemia and uremia)

5. Participation in another clinical trial or use of any other drug within the previous month

6. Recent use of ACEIs or ARBs except losartan in the past 1 month

7. Any excessive consumption of alcohol or any consumption of psychoactive substances, drug abuse or drug dependence during the past 5 years

8. According to the researcher's judgment, as some other diseases or conditions reduce the possibility of enrollment or complicate the enrollment, such as frequent changes of jobs and an unstable living environment, which may easily lead to loss of visits

\section{Randomization and concealment}

A specific randomization sequence will be computer generated by a computerized random number generator from an independent clinical research organization (CRO) of the Institute of Clinical Medicine of the Chinese Academy of Chinese Medical Sciences, which is not involved in the study. All eligible patients will be randomized to the experimental group or the control group at a 1:1 ratio. An independent non-investigator will protect the concealment list. The medical information will be confidential and not be available to any investigator for the duration of the study. If a medical emergency occurs, the individual's randomization code and group allocation can be identified.

\section{Blinding}

This study will be designed as double-blind. Not only are subjects and investigators blinded in these trials, but drug administration, statisticians and curative evaluators are also masked. Treatment allocation will be uncovered after completion of the study. In addition, TSF and placebo cannot be distinguished by their taste, smell and appearance. After production, study drugs will be packaged and transferred to numbered bottles by designated pharmacists in accordance with the randomized list. No one can tell the difference except the person who is in charge of concealment.

\section{Intervention}

All subjects in the two groups will receive the conventional treatment, including oral losartan $(50 \mathrm{mg} /$ once a day), diet, exercise and oral medicine, to ensure access to steady levels of blood glucose, blood lipids and blood pressure, based on the recommendations of the American Diabetes Association guidelines [7] and Treatment of Chinese Diabetes Society guidelines [8]. Subjects will be randomly assigned to receive placebo $(6 \mathrm{~g} / \mathrm{bag}$ twice per day) or Tangshan Formula $(6 \mathrm{~g} / \mathrm{bag}$ twice per day) by specified sequence number from the central randomization system. The treatment will last for 24 consecutive weeks.

\section{Outcomes}

\section{Primary outcomes}

Urinary microalbumin (MAlb) is a critical indicator for the diagnosis of early renal impairment in diabetes mellitus. For early-stage DKD, the urinary microalbumin-creatinine ratio (ACR) will be a primary evaluation index. The change in ACR from baseline will be evaluated between the two groups, and the ACR will be compared at 
baseline and treatment endpoint (24 weeks) in each group.

\section{Secondary outcomes}

1. Compare the ratio of progression into the clinical proteinuria period after intervention between the two groups

2. Urine microalbumin-negative conversion rate: the ratio of normal urinary microalbumin $(<20 \mu \mathrm{g} / \mathrm{min})$ compared between the two groups

3. Change in GFR: change in the D-value and ratio of glomerular filtration rate (GFR) before and after treatment compared between the two groups. GFR calculated using the simplified MDRD formula: GFR $\left(\mathrm{ml} / \mathrm{min} \cdot 1.73 \mathrm{~m}^{2}\right)=186 \times$ serum creatinine $1.154 \times$ age $-1.154 \times[$ female $\times 0.742] \times[$ Chinese $\times$ 1.233]

4. Doubling rate of baseline creatinine value compared between the two groups after intervention

Safety assessment Adverse events (AEs) will be continuously monitored for 24 weeks at the beginning and end of the study period, and the incidence of AEs will be evaluated at each visit, including vital signs, ECG, liver function, renal function, routine blood examination, routine urinalysis and routine stool examination. In addition, AEs, such as signs and symptoms and other ailments, will also be documented truthfully at every study visit, including the occurrence time, severity, duration, effective measures and transfer. Each $\mathrm{AE}$ associated with the intervention drugs will be classified as mild, moderate and severe. Severe AEs will need to be submitted to the Principal Investigator and the ethics committee within $24 \mathrm{~h}$. All AEs will be properly resolved. Criteria for the severity of adverse events' detailed requirements are as follows:

- Mild: mild discomfort, subjects can endure no treatment, no special treatment is required, no effect on subjects' recovery

- Moderate: moderate discomfort, unbearable to the subject, requiring special treatment, has a direct impact on the subject's recovery

- Severity: severe discomfort, life-threatening, fatal or disabling, immediate emergency treatment is required

\section{Study visits and assessment}

The intervention cycle will be 24 weeks, including a runin period. After the research commences, visits will occur every 4 weeks during the study period. An overview of specific measurements and time points for data collection is presented in Table 1.

\section{Quality control data collection}

To maintain the high quality of this trial and ensure its adherence to the protocol, all investigators and drug administrators participating in the research will be trained rigorously based on a standardized operation practice (SOP) manual. Withdrawals or dropped visits will also need to be explained on case report forms (CRFs). All data will be documented on a standardized CRF and instantly recorded in the database via the ClinResearch Electronic Data Capture System (http://www.tcmcec.net/crivrs/). The monitor will review the CRFs, check the inclusion, exclusion and withdrawal criteria, as well as ensure information on the CRFs is in accordance with those in the source medical records. Original CRFs will be reserved at the research center for 5 years after completion of the study. The validity and authenticity of the multicenter trial will be guaranteed by establishing three committees, including the clinical trial guidance committee, the data and safety monitoring board and the outcome evaluation committee, each respectively being responsible for the trial design and executing the process, monitoring the data collection process to control its quality and evaluating the outcomes. The flow diagram of enrollment, interventions and assessments is shown in Fig. 1.

\section{Sample size}

The sample size was estimated according to the relevant data from the losartan study published in New England Journal of Medicine [32, 33]. These results showed that the proportion of patients with the ACR reduced by $50 \%$ or more in the losartan group was $12.5 \%$ and the preliminary study of TSF data manifested that TSF as an addon study can improve $50 \%$ of patients. The estimated proportion of patients with the ACR reduced by $50 \%$ or more was $18.7 \%$. The estimated sample size formula was tested using the hypothesis of two population rates:

$$
n=\frac{[u \alpha \sqrt{2 p(1-p)}+u \beta \sqrt{p 1(1-p 1)+p 2(1-p 2)}]^{2}}{(p 1-p 2)^{2}}
$$

where $n$ is the sample size; $p_{1}$ and $p_{2}$ are the sample rates, and $p=\left(p_{1}+p_{2}\right) / 2$ is the sample average rate; and $\alpha$ is the type 1 error and $\beta$ is the type 2 error, while $u_{\alpha}$ and $u_{\beta}$ are the locus of the corresponding standard normal distribution. According to the unilateral test, $u_{\alpha}=1.64485$ and $u_{\beta}=0.84162$, and substituting these into the formula presents $n=274.46$. Therefore, 275 patients were needed in each group. Considering a dropout rate of no more than $15 \%$, the final sample size was estimated to be 632 in total. 
Table 1 Measurement items and point of data capture

\begin{tabular}{|c|c|c|c|c|}
\hline Visit project & $\begin{array}{l}\text { Screening period/ } \\
\text { baseline }\end{array}$ & Visits $1-2,4-5$ & Visit 3 & Visit 6 \\
\hline Visiting time & -7 to 0 days & $\begin{array}{l}\text { Medication } \\
4,8,16,20 \text { weeks } \pm 7 \\
\text { days }\end{array}$ & $\begin{array}{l}\text { Medication } \\
12 \text { weeks } \pm 7 \\
\text { days }\end{array}$ & $\begin{array}{l}\text { Medication } \\
24 \text { weeks } \pm 7 \\
\text { days }\end{array}$ \\
\hline \multicolumn{5}{|l|}{ Collect basic medical history } \\
\hline Sign informed consent & $\times$ & & & \\
\hline Fill in general information & $\times$ & & & \\
\hline Medical history and treatment history & $\times$ & & & \\
\hline Determine inclusion and exclusion criteria & $\times$ & & & \\
\hline Vital signs & $\times$ & $\times$ & $\times$ & $x$ \\
\hline Physical examination & $\times$ & $\times$ & $\times$ & $\times$ \\
\hline Comorbidity and medication records & $\times$ & $x$ & $x$ & $\times$ \\
\hline \multicolumn{5}{|l|}{ Monitoring and inspection } \\
\hline Urine pregnancy test & $\times$ & & & \\
\hline Glycosylated hemoglobin, blood lipids & $\times$ & & $\times$ & $\times$ \\
\hline Fasting blood sugar and blood pressure & $x$ & $x$ & $x$ & $x$ \\
\hline \multicolumn{5}{|l|}{ Effectiveness observation } \\
\hline Urinary microalbumin-creatinine ratio & $x$ & $x$ & $x$ & $x$ \\
\hline Urinary microalbumin excretion rate & $\times$ & $\times$ & $x$ & $\times$ \\
\hline Serum creatinine & $\times$ & $x$ & $x$ & $\times$ \\
\hline Glomerular rate filtration & $x$ & $x$ & $x$ & $\times$ \\
\hline $\begin{array}{l}\text { 24-h urinary microalbumin and 24-h urinary microalbumin } \\
\text { quantification }\end{array}$ & $x$ & & $x$ & $x$ \\
\hline \multicolumn{5}{|l|}{ Physico-chemical examination } \\
\hline Routine blood test and routine urine test & $\times$ & & $\times$ & $x$ \\
\hline Stool routine examination & $\times$ & & $x$ & $\times$ \\
\hline Vital signs & $\times$ & $x$ & $x$ & $x$ \\
\hline ECG, liver and kidney function & $\times$ & & $x$ & $x$ \\
\hline Adverse event & & $x$ & $x$ & $x$ \\
\hline \multicolumn{5}{|l|}{ Other work } \\
\hline Random grouping & $\times$ & & & \\
\hline Distribute drug and patient journal cards & $\times$ & $x$ & $x$ & \\
\hline Recovery drugs, quantity statistics & & $\times$ & $\times$ & $\times$ \\
\hline Recycle patient's diary card & & $\times$ & $\times$ & $\times$ \\
\hline End of study summary & & & & $\times$ \\
\hline
\end{tabular}

ECG electrocardiogram

\section{Statistical analysis}

The statistical analysis of this study was completed by an independent statistician, and the detailed statistical analysis plan was formulated separately by a statistician before the inception of this trial and determined with the Principal Investigator. Three analysis sets will be used for assessment of this study: the intention-to-treat set (ITT), the per-protocol analysis set (PPS) and the safety analysis set (SAS). The ITT and PPS will be used to appraise the efficacy of TSF. If any given case exists missing a critical variable, the last observation used as the final results will be carried forward to the final data. The changes of urinary microalbumin-creatinine ratio, urinary microalbumin, GFR, creatinine and baseline information will be present after treatment. A paired $t$ test or the Wilcoxon signedrank test will be employed to compare each group. Changes relative to baseline after treatment will be compared between groups using the $t$ test or the Wilcoxon rank-sum test. $P<0.05$ will be deemed to show a statistically significant difference. 


\section{Bias analysis}

The main evaluation outcome of this trial is the rate of progression from microalbuminuria into clinical proteinuria, which is very objective. Although ACEI and ARB drugs have achieved a recognized efficacy internationally, a certain proportion of patients still cannot delay the progression of DKD even using these two drugs. Therefore, the bias factors affecting the outcome evaluation include three aspects: blood glucose level; ACEI and/or $\mathrm{ARB}$ use; and laboratory testing error for urine microalbuminuria.

These factors are solved as follows: for the blood glucose level, the consistency of this factor in the two groups is ensured due to the random and double-blind study design method; subjects only take losartan and no other ARB or/and ACEI drugs; and central laboratories are used for the main outcomes.

\section{Discussion}

DKD is part of the systemic microangiopathy and glomerular sclerosis caused by diabetes. In European and American countries, DKD is the primary cause of renal replacement therapy, accounting for about $1 / 2$. It is the second common cause of ESRD in China after glomerular disease [1]. Compared to CKD from non-diabetic causes, DKD develops more rapidly into ESRD [34, 35]. It is urgent to seek an effective preventive measure to delay DKD. CHMs have been common in treating DM [36-41] and its complications [24, 42, 43], including DKD. In modern times, many patients have turned to Chinese herbal medicine for treatment as a complementary and necessary combination-drug therapy for kidney disease in China due to its fewer adverse reactions and more effective interventions. Currently, research from Taiwan has demonstrated that patients with CKD who used CHM had a significantly reduced ESRD risk (60\%) [44]. Relevant clinical observations have shown that TSF appears to prevent further development and deterioration of the disease, which includes the reduction of urinary albumin and normalization of the glomerular filtration rate [45-47]. Potential mechanisms have proved that herbal medicine could regulate oxidative stress, which is well recognized to play a significant role in the worsening of DKD [45, 48, 49]. However, there was less clinical research on delaying the progression of DKD, especially in the early stage as the only period of reversing kidney lesions [50]. This work has the potential function to delay the development of DKD. Therefore, we are implementing this study to evaluate the efficacy and safety of TSF treatment for DKD. If successful, this work will provide evidence-based medical evidence for a therapeutic approach to delaying the progress of DKD.

\section{Trial status}

Patient recruitment began in May 2018 and was expected to be completed in May 2022. At the time of manuscript submission, 95 patients had been recruited and the estimated time of recruitment completion is May 2020. Currently, we are still recruiting participants. Protocol version number 20,160,718 protocol02 (date 18 October 2016).

\section{Supplementary information}

Supplementary information accompanies this paper at https://doi.org/10. 1186/s13063-019-3821-6.

Additional file 1. SPIRIT 2013 Checklist: Recommended items to address in a clinical trial protocol and related documents.

\section{Abbreviations \\ ACEl: Angiotensin-converting enzyme inhibitor; ACR: Albumin-to-creatinine ratio; $\mathrm{AE}$ : Adverse event; $\mathrm{ARB}$ : Angiotensin II receptor blocker; $\mathrm{CHM}$ : Chinese herbal medicine; CKD: Chronic kidney disease; CRF: Case report form; CRO: Clinical research organization; DKD: Diabetic kidney disease; ESKD: End- stage kidney disease; GFR: Glomerular filtration rate; ITT: Intention-to-treat set; KDIGO: Kidney Disease Improving Global Outcomes; MAlb: Urinary microalbumin; NKF-KDOQI: National Kidney Foundation Kidney Disease Outcomes Quality Initiative; PPS: Per-protocol analysis set; RAAS: Renin- angiotensin-aldosterone system; SAS: Safety analysis set; SCr: Serum creatinine; SOP: Standardized operation practice; TSF: Tangshan Formula; UAER: Urinary microalbumin excretion rate}

\section{Acknowledgements}

The authors thank the State Administration of Traditional Chinese Medicine in China for supporting the study and give heartfelt thanks to all participants in this trial and the independent statistician.

\section{Authors' contributions}

DJ drafted the protocol. F-ML, QN and X-LT revised the protocol. W-JH, QB, $Y-n Y, F Y$ and $M-Z Z$ recruited the patients. All of the authors participated in the design and read and approved the final manuscript.

\section{Authors' information}

$X-L T$ is a principal investigator at China Academy of Chinese Medical Sciences. F-ML is researcher in Good Clinical Practice at Guang An Men Hospital. QN is head of the Endocrinology Department at Guang An Men Hospital.

\section{Funding}

The project is sponsored by the State Administration of Traditional Chinese Medicine (SATCM) in China (Grant number 201507001-11). During the whole research process, SATCM supports all of the research funding for the research design, data collection, analysis, manuscript writing and page charges.

\section{Ethics approval}

The Ethics Committee of Guang An Men Hospital of the China Academy of Chinese Medical Science has approved this trial for the participating centers (No. 2016-093-KY-01) and an informed consent form must be obtained before randomization.

Consent for publication

All participants have provided consent to share their individual medical information.

Competing interests

The authors declare that they have no competing interests. 


\section{Author details}

'Guang'anmen Hospital, Chinese Academy of Chinese Medical Sciences, Beijing 100053, China. ${ }^{2}$ Beijing University of Chinese Medicine, Beijing 100029, China.

\section{Received: 1 June 2019 Accepted: 22 October 2019}

\section{Published online: 21 December 2019}

\section{References}

1. United States Renal Data System. International comparisons. In: United States Renal Data System. 2014 USRDS annual data report: epidemiology of kidney disease in the United States. Bethesda: National Institutes of Health, National Institute of Diabetes and Digestive and Kidney Diseases; 2014. p. 188-210.

2. King P, Peacock I, Donnelly R. The UK Prospective Diabetes Study (UKPDS): clinical and therapeutic implications for type 2 diabetes. Br J Clin Pharmacol. 1999;48:643-8. https://doi.org/10.1046/j.1365-2125.1999.00092.x.

3. Dean J. Organising care for people with diabetes and renal disease. J Ren Care. 2012;38 Suppl 1:23-9. https://doi.org/10.1111/j.1755-6686.2012.00272.x.

4. Ahmad J. Management of diabetic nephropathy: recent progress and future perspective. Diabetes Metab Syndr. 2015;9(4):343-58

5. Cho NH, Shaw JE, Karuranga S, et al. IDF Diabetes Atlas: global estimates of diabetes prevalence for 2017 and projections for 2045. Diab Res Clin Pract. 2018;138:271.

6. Li R, Bilik D, Brown MB, et al. Medical costs associated with type 2 diabetes complications and comorbidities. Am J Manag Care. 2013;19(5):421 (PMID: 23781894).

7. American Diabetes Association. Glycemic targets: standards of medical care in diabetes—2018. Diabetes Care. 2018;41(Supplement 1):S55-64.

8. Jia W, Weng J, Zhu D, et al. Standards of medical care for type 2 diabetes in China 2019. Diabetes Metab Res Rev. 2019:e3158.

9. Hajhosseiny R, Khavandi K, Jivraj N, Mashayekhi S, Goldsmith DJ, Malik RA. Have we reached the limits for the treatment of diabetic nephropathy? Expert Opin Investig Drugs. 2014;23(4):511-22.

10. Dhodi JB, Mestry SN, Juvekar AR. Diabetic nephropathy-genesis, prevention, and treatment. Int J Pharm Pharm Sci. 2014;6(9):42-7 (Google Scholar).

11. Mogensen CE. Microalbuminuria predicts clinical proteinuria and early mortality in maturity-onset diabetes. N Engl J Med. 1984;310(6):356-60.

12. Viberti GC, Hill RD, Jarrett RJ, et al. Microalbuminuria as a predictor of clinical nephropathy in insulin-dependent diabetes mellitus. Lancet. 1982; 1(8287):1430.

13. Marre M, Chatellier $\mathrm{G}$, Leblanc $\mathrm{H}$, et al. Prevention of siabetic nephropathy with enalapril in normotensive diabetics with microalbuminuria. BMJ. 1988 297(6656):1092-5

14. Parving $\mathrm{HH}$, Persson $\mathrm{F}$, Lewis JB, et al. Aliskiren combined with losartan in type 2-diabetes and nephropathy_-secondary publication. N Engl J Med. 2008:358(23):2433.

15. Brenner BM, Cooper ME, De ZD, et al. Effects of losartan on renal and cardiovascular outcomes in patients with type 2 diabetes and nephropathy. N Engl J Med. 2001;11(1):861-9.

16. Tang JL, Liu BY, Ma KW. Traditional Chinese medicine. Lancet. 2008; 372(9654):1938-40.

17. Tian J, Jin D, Qi B, et al. Evidence and Potential Mechanisms of Traditional Chinese Medicine for the Treatment of Type 2 Diabetes: A Systematic Review and Meta-analysis[J]. Diabetes, Obesity and Metabolism. 2019.

18. Liu J, Henkel T. Traditional Chinese medicine (TCM): are polyphenols and saponins the key ingredients triggering biological activities? Curr Med Chem. 2002:9(15):1483-5.

19. Letasiová S, Jantová S, Cipák L, et al. Berberine-antiproliferative activity in vitro and induction of apoptosis/necrosis of the U937 and B16 cells. Cancer Lett. 2006;239(2):254.

20. Bang JY, Kim KS, Kim EY, et al. Anti-angiogenic effects of the water extract of HangAmDan (WEHAD), a Korean traditional medicine. Sci China Life Sci. 2011;54(3):248-54

21. Wedge DE, Gao Z, Tabanca N, et al. Chemical composition and biological activities of two Angelica essential oils from China. Planta Med. 2009:75(4):422

22. Wang FL, Tang LQ, Yang F, et al. Renoprotective effects of berberine and its possible molecular mechanisms in combination of high-fat diet and lowdose streptozotocin-induced diabetic rats. Mol Biol Rep. 2013;40(3):2405-18.
23. Zhao XF, Su SJ, Yun G, et al. Mortality and recurrence of vascular disease among stroke patients treated with combined TCM therapy. J Tradit Chin Med. 2012;32(2):173.

24. Chen $H$, Jing $G$, Zhao $X$, et al. Retrospective analysis of the overt proteinuria diabetic kidney disease in the treatment of modified Shenzhuo formula for 2 years. Medicine. 2017:96(12):e6349.

25. Guo J, Hongdong C, Xiyan Z, et al. Diabetic kidney disease treated with a modified Shenzhuo formula derived from Traditional Chinese Medicine: a case report. J Tradit Chin Med. 2017;37(6):854-61.

26. Tian J, Zhao L, Zhou Q, et al. Efficacy of Shenzhuo formula on diabetic kidney disease: a retrospective study. J Tradit Chinese Med. 2015;35(5):528.

27. Moher D, Hopewell S, Schulz KF, et al. CONSORT 2010 explanation and elaboration: updated guidelines for reporting parallel group randomised trials. J Clin Epidemiol. 2010;63(8):e1-e37.

28. Boutron I, Altman DG, Moher D, et al. CONSORT Statement for Randomized Trials of Nonpharmacologic Treatments: a 2017 update and a CONSORT extension for nonpharmacologic trial abstracts. Ann Intern Med. 2017;167(1): 40-7.

29. Chan A W, Tetzlaff J M, Gøtzsche P C, et al. SPIRIT 2013 explanation and elaboration: guidance for protocols of clinical trials[J]. BMJ. 2013;346:e7586

30. National Kidney Foundation. KDOQI clinical practice guideline for diabetes and CKD: 2012 update. Am J Kidney Dis. 2012;60(5):850-86.

31. Levin A, Stevens PE, Bilous RW, et al. Kidney Disease: Improving Globa Outcomes (KDIGO) CKD Work Group. KDIGO 2012 clinical practice guideline for the evaluation and management of chronic kidney disease. Kidney Int Suppl. 2013;3(1):1-150 (Google Scholar).

32. Fried LF, Emanuele N, Zhang JH, Brophy M, Conner TA, Duckworth W, et al. Combined angiotensin inhibition for the treatment of diabetic nephropathy. N Engl J Med. 2013;369(20):1892-903.

33. Montero de Francisco A, García-Luque A, Fernandez M, Puerro M. Side effects of angiotensin converting enzyme inhibitors and angiotensin II receptor antagonists: are we facing a new syndrome. Am J Cardiol. 2012; 110(10):1552-3.

34. Mogensen CE, Christensen CK, Vittinghus E. The stages in diabetic renal disease. With emphasis on the stage of incipient diabetic nephropathy. Diabetes. 1983:32(Suppl 2):64-78.

35. Piccoli GB, Grassi G, Cabiddu G, Nazha M, Roggero S, Capizzi I, et al. Diabetic kidney disease: a syndrome rather than a single disease. Rev Diabet Stud. 2015;12(1-2):87-109.

36. Tong $X, X u$ J, Lian F. Structural alteration of gut microbiota during the amelioration of human type 2 diabetes with hyperlipidemia by metformin and a traditional Chinese herbal formula: a multicenter, randomized, open label clinical trial. Mbio. 2018;9(3):3-7.

37. $Y u X, X u L$, Zhou Q, et al. The efficacy and safety of the Chinese herbal formula, JTTZ, for the treatment of type 2 diabetes with obesity and hyperlipidemia: a multicenter randomized, positive-controlled, open-label clinical trial. Int J Endocrinol. 2018;2018(1):9519231.

38. Tian J, Lian F, Yu X, et al. The efficacy and safety of Chinese herbal decoction in type 2 diabetes: a 5-year retrospective study. Evid Based Complement Alternat Med. 2016;2016(9):5473015.

39. Lian F, Tian J, Chen $\mathrm{X}$, et al. The efficacy and safety of Chinese herbal medicine Jinlida as add-on medication in type 2 diabetes patients ineffectively managed by metformin monotherapy: a double-blind, randomized, placebo-controlled, multicenter trial. PLoS One. 2015;10(6):e0130550.

40. Xu J, Lian F, Zhao L, et al. Structural modulation of gut microbiota during alleviation of type 2 diabetes with a Chinese herbal formula. ISME J. 2015; 9(3):552-62

41. Ji L, Tong $X$, Wang $H$, et al. Efficacy and safety of traditional Chinese medicine for diabetes: a double-blind, randomised, controlled trial. PLoS One. 2013:8(2):e56703.

42. He L, Wang H, Gu C, et al. Administration of traditional Chinese blood circulation activating drugs for microvascular complications in patients with type 2 biabetes mellitus. J Diabetes Res. 2016;2016(10):1-9.

43. Lian F, Wu L, Tian J, et al. The effectiveness and safety of a danshencontaining Chinese herbal medicine for diabetic retinopathy: a randomized,double-blind,placebo-controlled multicenter clinical trial. J Ethnopharmacol. 2015;164:71-7.

44. Lin MY, Chiu YW, Chang JS, Lin HL, Lee CT, Chiu GF, Kuo MC, Wu MT, Chen HC, Hwang SJ. Association of prescribed Chinese herbal medicine use with risk of end-stage renal disease in patients with chronic kidney disease. Kidney Int. 2015;88(6):1365-73. 
45. Fan $\mathrm{XM}$, Huang $\mathrm{CL}$, Wang $\mathrm{YM}$, et al. Therapeutic effects of Tangshen Formula on diabetic nephropathy in $\mathrm{db} / \mathrm{db}$ mice using cytokine antibody array. J Diabetes Res. 2018;2018(3):1-10.

46. Zhao TT, Sun SF, Zhang HJ, et al. Therapeutic effects of Tangshen Formula on diabetic nephropathy in rats. PLoS One. 2016;11(1):e0147693.

47. Huang M, Zhu C, Liang QL, et al. Effect of Tangshen Formula on phospholipids metabolism in diabetic nephropathy patients. Acta Pharmaceutica Sinica. 2011;46(7):780 (PMID:22010346).

48. Miranda-Díaz AG, Pazarín-Villaseñor L, Andrade-Sierra J. Oxidative stress in diabetic nephropathy with early chronic kidney disease. J Diabetes Res. 2016;2016(1):4-6.

49. Kashihara N, Haruna Y, Kondeti VK, et al. Oxidative stress in diabetic nephropathy. Curr Med Chem. 2010;17(34):4256-69.

50. Pathak JV, Dass EE. A retrospective study of the effects of angiotensin receptor blockers and angiotensin converting enzyme inhibitors in diabetic nephropathy. Indian J Pharmacol. 2015;47(2):148-52.

\section{Publisher's Note}

Springer Nature remains neutral with regard to jurisdictional claims in published maps and institutional affiliations.

Ready to submit your research? Choose BMC and benefit from:

- fast, convenient online submission

- thorough peer review by experienced researchers in your field

- rapid publication on acceptance

- support for research data, including large and complex data types

- gold Open Access which fosters wider collaboration and increased citations

- maximum visibility for your research: over $100 \mathrm{M}$ website views per year

At BMC, research is always in progress.

Learn more biomedcentral.com/submissions 